Mens

revue d'histoire intellectuelle de l'Amérique française

\title{
Yvan Lamonde et Denis Saint-Jacques (dir.). 1937 : un tournant culturel, Québec, Les Presses de l’Université Laval, 2009, 371 p.
}

\section{Jonathan Livernois}

Volume 11, numéro 1, automne 2010

URI : https://id.erudit.org/iderudit/1023345ar

DOI : https://doi.org/10.7202/1023345ar

Aller au sommaire du numéro

Éditeur(s)

Centre de recherche en civilisation canadienne-française

ISSN

1492-8647 (imprimé)

1927-9299 (numérique)

Découvrir la revue

Citer ce compte rendu

Livernois, J. (2010). Compte rendu de [Yvan Lamonde et Denis Saint-Jacques (dir.). 1937 : un tournant culturel, Québec, Les Presses de l’Université Laval, 2009, 371 p.] Mens, 11(1), 124-127. https://doi.org/10.7202/1023345ar d'utilisation que vous pouvez consulter en ligne.

https://apropos.erudit.org/fr/usagers/politique-dutilisation/ 
de mémoire et de sa signification historique (Saint-Denis-surRichelieu) à une cellule sociale porteuse de mémoire (la parenté), à la création, par les arts, d'espaces où s'inscrit la mémoire francophone, les contributions à ce collectif montrent que les francophones du pays possèdent leurs lieux et leur mémoire propres et ne se représentent pas tous le passé de la même façon. Puisqu'il demeure impossible de décrire une mémoire francophone qui soit collective, c'est-à-dire portée simultanément par tous les francophones du Canada, c'est bel et bien dans toute sa diversité, mais en dépit de sa fragmentation, que la francophonie canadienne parviendra à s'inscrire dans la durée.

- Julie Léger

Département d'histoire

Université d'Ottawa

\section{Yvan Lamonde et Denis Saint-Jacques (dir.). 1937 : un tournant culturel, Québec, Les Presses de l'Université Laval, 2009, 371 p.}

On s'est souvent intéressé à la débâcle de 1960 ; de plus en plus, on s'attache aux périodes de gel et de dégel qui la précédèrent. Ainsi paraissaient récemment les actes d'un colloque, tenu en novembre 2007 à l'Université du Québec à Montréal, consacré à l'année 1937. L'hypothèse des coresponsables, Yvan Lamonde et Denis SaintJacques, voulait " que l'année 1937 soit dans nombre de domaines un moment où des nœuds culturels, intellectuels, esthétiques ou idéologiques deviennent irrécusables et se dénouent dans certains cas » (p. vir). Lors du colloque de 1985 sur l'avènement de la modernité culturelle au Québec, Lamonde avait déjà montré que les années 1930, par une valorisation de l'expression, par la volonté d'être de son temps et par le développement d'une subjectivité forte, avaient tout d'un tournant de notre histoire culturelle. Vingt-deux ans plus 
tard, de nombreux spécialistes de diverses disciplines ont exemplifié et confirmé cette hypothèse.

Le pari était audacieux : réussir à montrer en quoi cette année 1937 est représentative d'une décennie elle-même décisive d'un point de vue culturel, et ce, tout en ne surinterprétant pas les événements qui la ponctuèrent. Cela dit, même si un événement n'eut qu'un retentissement mineur, voire inexistant en 1937, il semble qu'il puisse aussi éclairer la suite des choses : on notera, en ce sens, la présentation que Mathieu Lapointe fait des lettres que le jeune Raoul Roy envoya en 1937 à l'abbé Lionel Groulx, témoignages qui n'ont d'intérêt véritable que parce que Roy, au début des années 1960, fut l'une des principales figures de l'indépendantisme socialiste. Le point de vue est original.

Du Deuxième Congrès de la langue française au Canada aux midinettes en grève, en passant par la caricature de Robert LaPalme, le théâtre, l'américanité, les chansons western, le cinéma d'Albert Tessier, mais aussi par Directives de Lionel Groulx, la visite de Malraux à Montréal et Séparatisme, doctrine constructive de Dostaler O'Leary, les vingt-trois collaborations sont diversifiées, sans toutefois réussir à tout embrasser, ce qui va de soi. Par contre, on aurait aimé lire davantage de réflexions sur les grèves de Sorel, sur la création de la Commission royale d'enquête des relations entre le Dominion et les provinces (commission Rowell-Sirois) et sur la mort d'Olivar Asselin, entre autres sujets. On aura aussi été étonné par un texte par trop impressionniste de Philippe Dubé consacré à François Hertel. Déception, finalement, quant à la place faite au centenaire des rébellions par Marie-Frédérique Desbiens, dont la présentation, un peu courte, ne nous a pas convaincu. On aurait souhaité que l'auteure revienne, par exemple, sur les réflexions de Lionel Groulx, dont «Une heure avec l'abbé Groulx à propos de "37" " constitue un bel aperçu. Elle aurait aussi pu s'intéresser au roman de François Hertel, Le beau risque (1939), dont l'action se déroule quelque temps avant les célébrations du centenaire. 
Malgré ces quelques réserves, 1937 : un tournant culturel est la somme de travaux qui sont généralement de haute tenue intellectuelle, encadrés par une introduction qui va droit au but et une double conclusion particulièrement efficace, qui permet de mettre de l'ordre dans les hypothèses disséminées au fil des pages. Il faut noter certaines collaborations particulièrement stimulantes qui répondent à l'appel lancé par Saint-Jacques et Lamonde. Nous avons déjà évoqué le parcours original de Raoul Roy, bien présenté par Mathieu Lapointe. Il faut rappeler le très beau texte de Vincent Charles Lambert, dont le style et la sensibilité sont à la mesure de son sujet : Hector de Saint-Denys Garneau. Il y a là une manière qui rappelle Pierre Nepveu. Nous pensons aussi à la collaboration d'Esther Trépanier, qui montre que l'art moderne au Canada (français), contrairement à celui des pays européens et des États-Unis, ne s'est pas fortement attaché au " mode de vie capitaliste et à la culture de masse " mais bien plutôt à " une certaine culture populaire " (p. 199). Rappelant le grand intérêt de nombreux critiques et peintres pour la peinture d'enfants, comme elle l'avait fait d'ailleurs dans son ouvrage Peinture et modernité au Québec, 1919-1939 (Nota bene, 1998), Trépanier propose une piste que nous considérons comme une invitation : "Il y aurait beaucoup à dire sur tout ce qui est en jeu dans les rapports qui s'établissent entre art moderne et arts "primitif", "traditionnel" ou "naif" qui permettrait aussi de mieux comprendre pourquoi c'est plutôt vers la culture populaire traditionnelle que vers la culture urbaine contemporaine que se tournent certains artistes québécois dans leur quête de modernité. " (p. 201) Une modernité originale, qui se construit en se retournant vers le passé? L'enfance et la naïveté, connotées positivement chez les peintres et les critiques, pour en finir avec «l'infantilisme " de la culture canadienne-française, dénoncé quelques années plus tard par de nombreux intellectuels comme Jean LeMoyne, Pierre Vadeboncœur et le père Ernest Gagnon? Voilà d'étranges directions de la modernité culturelle auxquelles le texte de Trépanier nous invite à réfléchir. 
Il faut aussi rappeler le propos profondément original de la collaboration de Laurier Lacroix, "Les barbares, nos premiers modernes ou "Comment New York a volé..." au secours de l'art moderne dans la province de Québec ». Le fait que ce texte soit aussi consacré à la modernité picturale laisse croire que la conviction que Pierre Vadeboncœur a développée au début des années 1960 était plutôt juste : les premiers pas de la modernité furent surtout, au Canada français, ceux de la peinture. Évoquant l'exposition de peintres populaires charlevoisiens à New York à la fin de l'année 1937, Lacroix montre le passage étonnant du monde rural à l'avant-garde artistique. Un lien, inusité, s'établit même entre cet art et celui des futurs automatistes; « le plaisir de peindre dont témoignaient les artistes de Charlevoix fut un encouragement et une leçon que reçurent les jeunes artistes à un moment crucial de leur adhésion à la modernité picturale " (p. 298). Comme Esther Trépanier, Laurier Lacroix tire un fil surprenant de la modernité : «C'est du milieu rural que vient une leçon propre à servir la cause des acteurs à l'avant-plan de la peinture moderne. " (p. 298) La conception de la modernité comme rupture totale s'étiole encore un peu plus ici. Ce n'est peut-être pas un hasard, d'ailleurs, si l'on a reproduit une toile de la Charlevoisienne Adéla Harvey (Le charroyage de l'eau, 1936) sur la page couverture du volume. Le sous-titre implicite des actes serait-il « La modernité par un retour ambivalent au passé»?

À tout prendre, 1937 : un tournant culturel permet non seulement de mettre à mal certains poncifs de la modernité, mais donne aussi un aperçu généreux de ce qu'une année peut révéler sur le Canada français, pris entre tradition et modernité. Le pari, risqué, a été remporté. On souhaite que d'éventuelles rencontres s'articulent autour d'autres années décisives. Il y en a beaucoup avant la débâcle.

- Jonathan Livernois Département de langue et littérature françaises Université McGill 